\title{
FAULT DETECTION AND ISOLATION OF AIRCRAFT AIR DATA/INERTIAL SYSTEM
}

\author{
D. Berdjag ${ }^{1}$, J. Cieslak ${ }^{2}$, and A. Zolghadri ${ }^{2}$ \\ ${ }^{1}$ LAMIH Lab, University of Valenciennes \\ Le Mont Houy, Valenciennes 59313, France \\ ${ }^{2}$ IMS Lab, University of Bordeaux \\ 351 Cours de la Libération, Talence Cedex 33405, France
}

\begin{abstract}
A method for failure detection and isolation (FDI) for redundant aircraft sensors is presented. The outputs of the concerned sensors are involved in the computation of flight control laws, and the objective is to eliminate any fault before propagation in the control loop when selecting a unique flight parameter among a set (generally, three) of redundant measurements. The particular case of an oscillatory failure is investigated. The proposed method allows an accurate FDI of erroneous sensor and computes a consolidated parameter based on the fusion of data from remaining valid sensors. The benefits of the presented method are to enhance the data fusion process with FDI techniques which improve the performance of the fusion when only few sources (less than three) are initially valid.
\end{abstract}

\section{INTRODUCTION}

The state-of-practice for aircraft manufacturers to diagnose guidance and control (G\&C) faults and obtain full flight envelope protection at all times is to provide high level of hardware redundancy in order to perform consistency tests and to ensure sufficient available control action. In the frame of future environmentallyfriendlier aircraft and structural design optimization, this work deals with a method of integrity control based on the processing of anemometric and inertial data in the Flight Control Computer (FCC). The FCC provides data used to compute a command (position order) to servocontrol each moving surface (Fig. 1). The data are acquired using an inertial acquisition system composed 


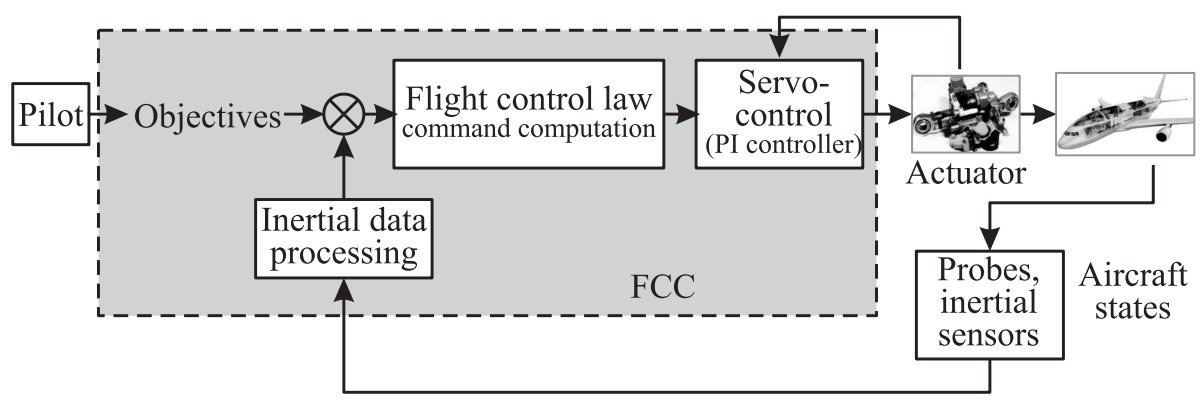

Figure 1 Flight control law computation

by several dedicated redundant units (usually, three). The FCC generally receives three redundant values of each flight parameter from the sensors and computes unique flight parameter value required for the flight control laws computation.

This specific data fusion processing, called "consolidation," classically consists of two simultaneous steps: from the three sources, selection of one unique and accurate parameter and in parallel, monitoring of each of the three independent sources to discard any failed source and to ensure that the selected value is correct. This overall "consolidation" processing selects the reliable flight parameters with the required accuracy by discarding any possible failed redundant source.

Current consolidation state-of-practice allows to be compliant with stringent regulations. However, for future aircraft structural design optimization, it could be required to avoid the propagation of oscillations even of smaller amplitudes.

Fault-tolerant management system checks the consistency of all sensor outputs to detect a failed source, typically by using a majority-voting or a weighted mean method [1] or soft-computing approaches [2]. The main advantages of this architecture are the design and integration simplicity [3]. Also, to provide safe operation, the architecture must have at least three valid sources, which means the fault tolerance could not be guaranteed after a single source failure: if a source fails, then it has to be removed from the fault tolerant architecture.

The sensor management system proposed in this work aims to solve the inherent issue of losing a source in a three sensor acquisition system or triplex, and to be still capable of a sufficient fault tolerant data acquisition. The issue is investigated for very specific oscillatory failures that may occur in the flight parameter sensors. The presented approach is based on a hierarchical FDI structure which takes in consideration the number of healthy sensors in 
the system. When more than two sensors are available, soft computing techniques are used for data consolidation. Otherwise, filter-based FDI approach is used to detect the failure node by node. The novelty in the method is the use of a particular residual generator similar to the harmonic filter developed in [4] to transform the fault signature in the residual from sinusoidal to steplike, greatly improving the detection rate for low amplitude oscillatory failures. The application is related to on-going research undertaken within the European ADDSAFE project [5] to assess the capability of efficient model-based fault detection and diagnosis (FDD) methods on realistic aircraft flight control systems.

This work is organized as follows:

- section 2 is devoted to problem formulation;

- section 3 presents the proposed structure based on a mixed data fusion / FDI method for consolidation;

- section 4 presents the simulation results obtained from a high-fidelity benchmark; and

- concluding remarks are discussed in the last section.

\section{PROBLEM FORMULATION}

One class of flight parameter sensor failures is additive oscillations appearing on output signals. These failures are referred as Combined Oscillatory Failure Cases (COFC) in contrast to Oscillatory Failure Cases (OFC) which impact only one control surface $[6,7]$. Combined oscillatory failure cases can impact several control surfaces. The measurements provided by the corrupted source could propagate through the control loop and may cause under some circumstances unwanted oscillations of the control surfaces. Thus, the corrupted sources need to be switched off as fast as possible.

Current industrial practices involve triplex voting schemes as in [8-10]. The basic principle, shown in Fig. 2, is to sort output signals and to give a 0.5 weighting to the source providing the median value of the parameter and a 0.25 weighting to the two other sources, then adding the results to obtain the value of the parameter. A threshold, centered around the obtained value, is used to detect the occurrence of a failure. When a COFC occurs, the corrupted source is detected when the provided measurement stays outside the threshold for a specific amount of time.

If only two sources are valid, the consolidation is performed by choosing the mean value of the two measurements. Notice the discontinuity when switching from vote-based consolidation to the mean-based one. If the difference between 


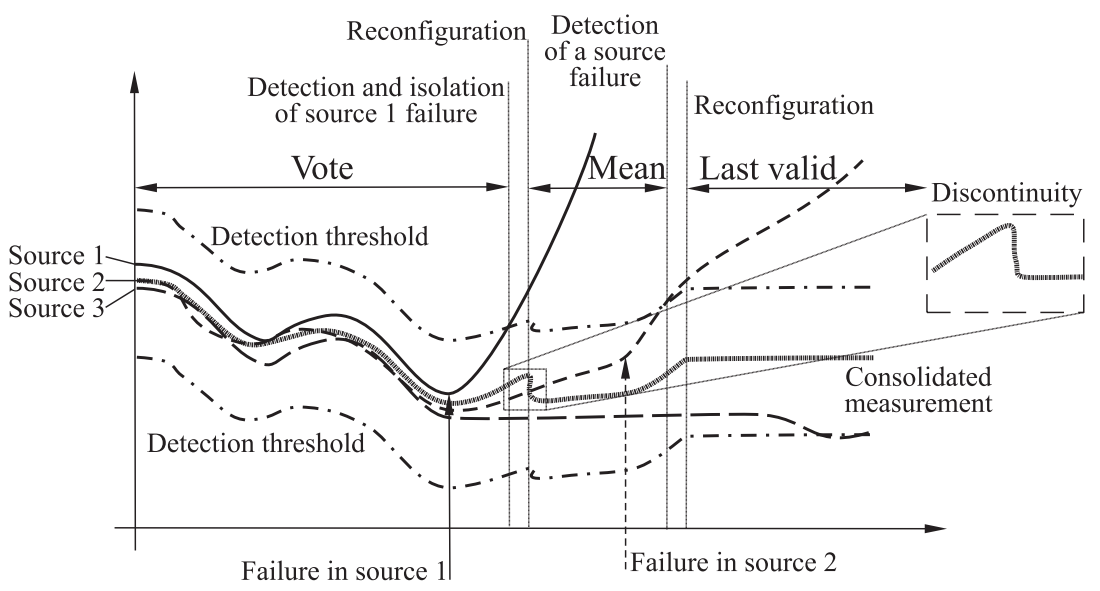

Figure 2 Triplex monitoring

the two signals is superior to a specified threshold, then the two sources are discarded and the last correct value of the parameter is used. On fault occurrence, the isolation is possible if initially the three sources are valid, and all measurement updates are lost when two sources are declared corrupted, since it cannot be decided which source remains valid. Also, thresholds must be chosen offline for all the possible flight scenarios, involving long and costly experiments. Finally, as it can be seen in Fig. 2, transients appear on the consolidated parameter.

The main objective of this work is to enhance the classical majority-vote triplex monitoring. Two failure cases are investigated: the first one starting with three valid sources initially (where the classical majority-based approach is appropriate) and the second one starting with two valid sources and a corrupted source initially (where the majority-based approach is impossible).

In order to improve the existing methodology on the first scenario and to extend failure isolation functionality on the second one, a mixed Data Fusion (DF) / FDI method is proposed in the following section.

\section{DETAILED DATA FUSION / FAULT DETECTION AND ISOLATION METHOD}

\subsection{General Structure}

The overall structure of the proposed method is shown in Fig. 3. There are two major components. The first one is the FDI module composed by residual generators and decision-making module, and the second component is the 


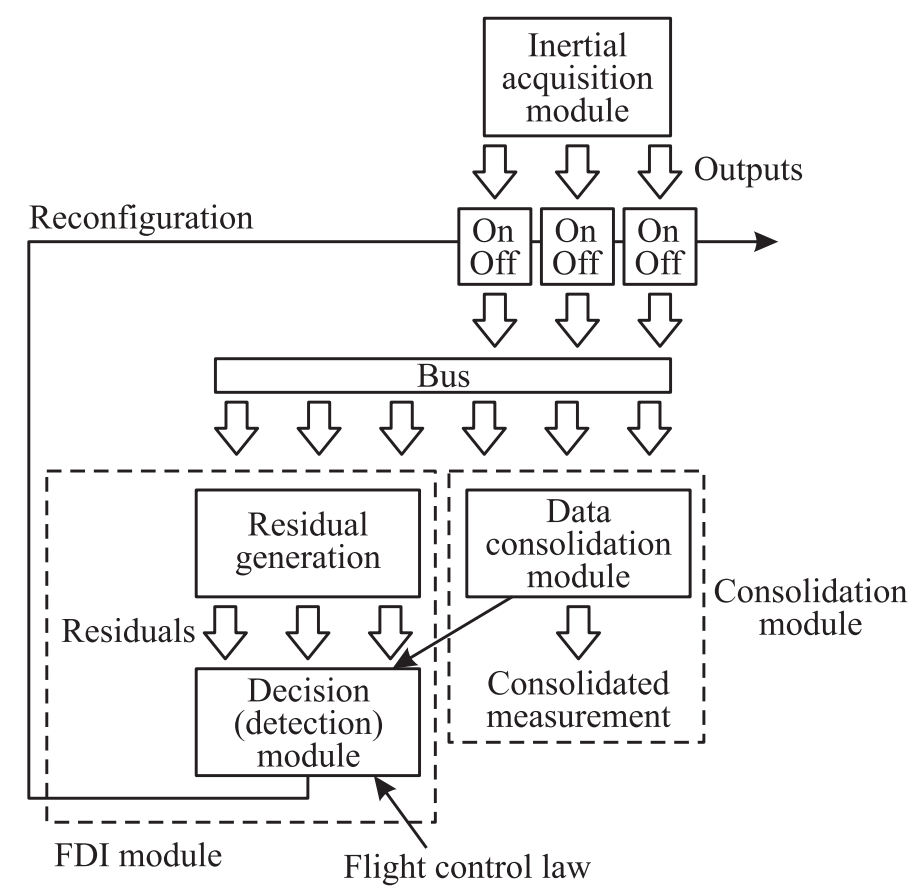

Figure 3 Overall structure of the proposed methodology

consolidation module. The role of the FDI module is to detect the corrupted source and to switch it off as soon as possible, while the consolidation module gives an accurate estimation of the parameter based on the measurement data provided by the valid sources. The knowledge of the flight control law is used to discriminate between the COFC and a possible (normal) pilot input, since the oscillation detection is performed separately on each sensor and the filtering will not discriminate between the "good" and the "bad" oscillations. The consolidation module provides also FDI functionality in the three valid sources scenario. In order to minimize the computation time, the FDI module is switched ON only when a sensor is declared corrupted, since when all the sources are valid, consolidation module provides sufficiently performant fault isolation.

\subsection{Consolidation Module}

The consolidation module is similar to the system proposed in [2]. The fusion between different sources is performed using a Fuzzy logic approach called Soft- 
voting. Each source is assigned a weight corresponding to the amount of trust it is credited, and the consolidated signal is the weighted average of all valid sources:

$$
S_{\text {vote }}=\sum_{i=1}^{n_{\text {valid }}} w_{i} S_{i}
$$

with $w_{i}$ representing the weight to the source $S_{i}$ and $n_{\text {valid }}$ corresponding to the number of valid sources. The weight $w_{i}$ is computed from the membership degree $\mu_{i} \in[0,1]$ assigned to each measurement:

$$
w_{i}=\frac{\mu_{i}}{\sum_{j=1}^{n_{\text {valid }}} \mu_{j}} .
$$

The computation of different values of $\mu_{i}$ is given in $[2,11]$. Each membership function is centered on the value provided by the corresponding source (Fig. 4) and used to determine the membership degree of the source, which is given by the largest membership degree $q_{j}$ of the remaining valid signals:

$$
\mu_{i}=\max _{i \neq j}\left(\mu_{i}\left(q_{j}\right)\right)
$$
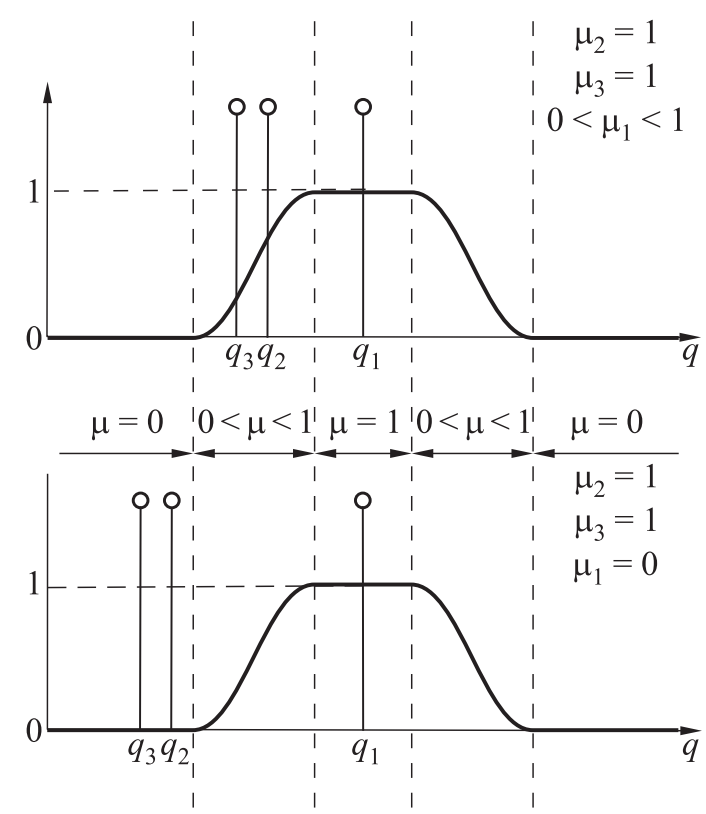

Figure 4 Membership degree computation 
As it can be seen, the majority voting concept is used in soft voting as it is used in conventional consolidation. The difference is the contribution of the measurement signals: in conventional scheme, the contribution of the potentially faulty signal is rejected from the start while in soft voting scheme, the contribution of the signal is reduced gradually before rejection. The direct consequence of the "rejection" vs. the "reduction" is the discontinuity of the consolidated mea-

Table 1 Soft monitoring procedure

\begin{tabular}{l} 
If $\mu_{i}=1$ then count $i=$ count $_{i}-1$ \\
If $0<\mu_{i}<1$ then count $i=$ count $_{i}$ \\
If $\mu_{i}=0$ then count count $_{i}+2$ \\
\hline
\end{tabular}
surement appearing when a failed source is switched off in the classical voting scheme. In the soft-voting based consolidation, the consolidated measurement remains smooth in all cases. The overall structure of the soft voting block is given in [11]. The monitoring component is based on a counter associated to each source and a maximum allowed threshold (Table 1).

Another very important point is the periodic nature of the failure. Using this complementary information, the detection can be performed by monitoring the periods between transitions from 1 to 0 of a membership degree $\mu_{i}$ as shown in
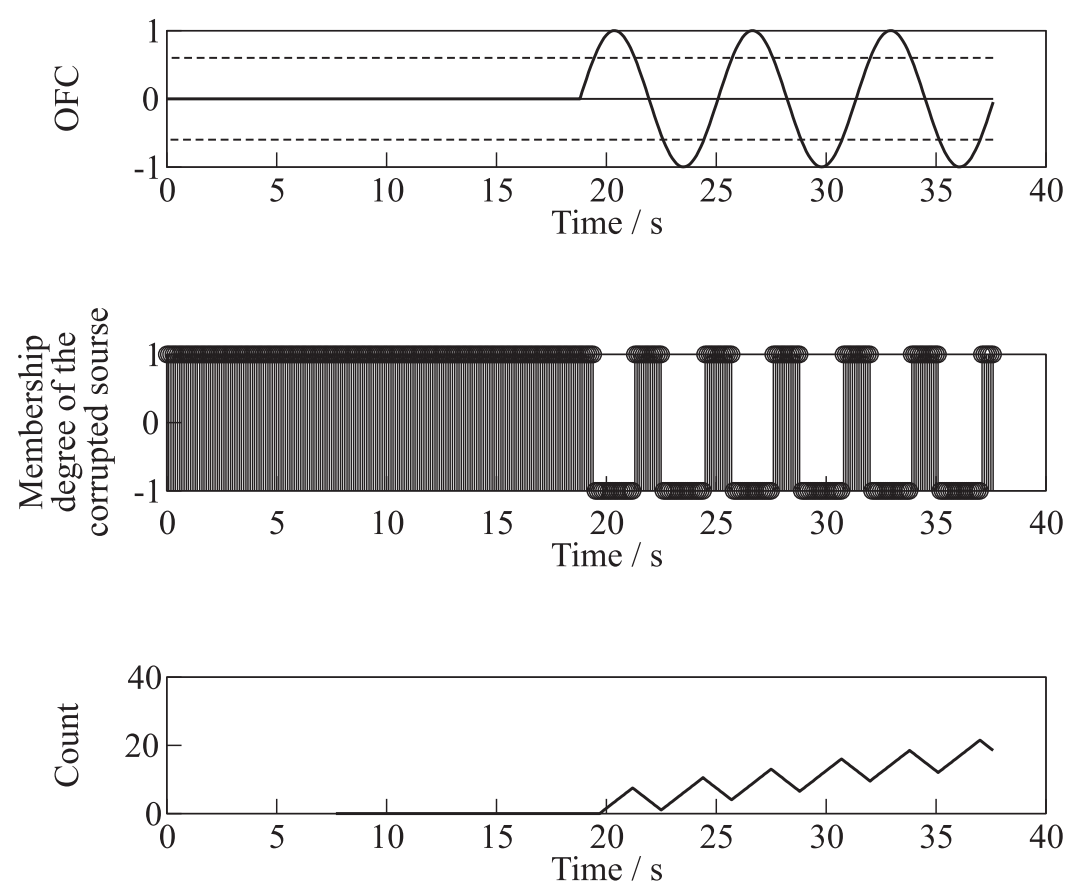

Figure 5 Oscillatory failure cases behavior 


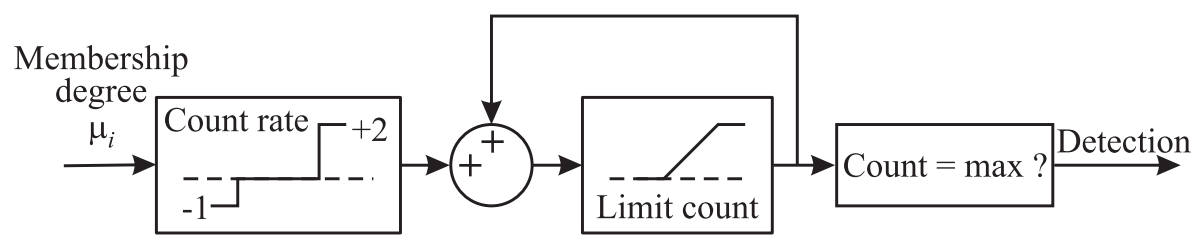

Figure 6 Soft monitor

Fig. 5. When four successive 1 to 0 transitions of $\mu_{i}$ show periodicity, an OFC is detected. Notice that the counter also can provide a detection for a large amplitude OFC. This is useful to detect a single OFC occurring in three valid sensors scenarios.

The overall scheme of the monitoring block is shown in Fig. 6. Since the counter rate is not a function of the difference between the consolidated value and the $i$ th measurement as in the conventional scheme, but a function of the difference between the different measurements, therefore, no transients occur when a source is switched off because its contribution to the consolidated parameter was already nil.

\subsection{Fault Detection and Isolation Module}

Since the only knowledge on the system is the measured outputs and the characteristics of the expected failures, signal processing based FDI methods can be preferred. The proposed FDI module is based on a particular characteristic of the harmonic filter proposed in [4]. For the considered problem, the parameter estimation functionality of this filter is useless; however, the filter is very sensitive to new harmonics appearing in input signal spectrum. This functionality will be used to design a selective residual generator.

The measured signals are noisy; so, the direct residual generation is difficult. Additional filtering component is considered in the form of a suboptimal steadystate filter similar to the filter developed in $[12,13]$.

Finally, an abrupt change detection method is used to perform COFC detection. In this work and because of harsh computer time constraints, a robust derivative estimator is used to amplify residual changes and threshold crossing detection. The thresholds are computed offline, in fault-free conditions.

\subsubsection{Kalman filtering}

Consider the following fault signal:

$$
f(t)=a \cos \left(\omega_{0} t+\phi\right)
$$


where $a, \omega_{0}$ and $\phi$ are, respectively, the amplitude, frequency, and phase of the sinusoidal signal $f(t)$. The corresponding state space model is

$$
\left.\begin{array}{c}
{\left[\begin{array}{c}
\dot{x}_{1} \\
\dot{x}_{2}
\end{array}\right]=\underbrace{\left[\begin{array}{cc}
0 & 1 \\
-\omega_{0}^{2} & 0
\end{array}\right]}_{A}\left[\begin{array}{l}
x_{1} \\
x_{2}
\end{array}\right] ;} \\
f=\underbrace{\left[\begin{array}{ll}
a & 0
\end{array}\right]}_{C} x+v
\end{array}\right\}
$$

where $v$ is the Gaussian noise. The Kalman Filter for the system described by Eqs. (2) is:

$$
\dot{\hat{x}}=A \hat{x}+H[f-C \hat{x}] .
$$

In [12], an appropriate value of $H$ for this model is given by

$$
H=2 \xi \omega_{0}\left[\begin{array}{l}
1 \\
0
\end{array}\right] .
$$

It is a reasonable choice leading to a suboptimal steady-state filter, with $\xi$ being a small constant and $a$ set to $a=1$. The final state-space equation of this filter is given by

$$
\dot{\hat{x}}=\left[\begin{array}{cc}
-2 \xi \omega_{0} & 1 \\
-\omega_{0}^{2} & 0
\end{array}\right] \hat{x}+2 \xi \omega_{0}\left[\begin{array}{l}
1 \\
0
\end{array}\right] f .
$$

This corresponds to a stable second order transfer function between the output and $\hat{x}_{1}$ :

$$
G(s)=\frac{\hat{X}_{1}}{F}=\frac{2 \xi \omega_{0} s}{s^{2}+2 \xi \omega_{0} s+\omega_{0}^{2}} .
$$

This simple system provides suboptimal performance when filtering sinusoidal signals around $\omega_{0}$ frequency, $\pm 5 \mathrm{~Hz}$, which fits rather well for the case of interest. However, should the OFC occur in a larger frequency band, the use of an extended Kalman filter must be considered, augmenting the state with the parameter $\Delta \omega_{0}$, representing the uncertainty on $\omega_{0}$.

\subsubsection{Residual generation}

A classical decision-making method in FDI is to check threshold crossing by the residual signal. An usual problem here is to determine appropriate threshold values in order to simultaneously maximize fault detection ratio and to minimize detection delay and false alarms rate. This problem has many possible solutions for fault signatures that induce abrupt changes in the system behavior (see, for instance, [14-17]. But for oscillatory failures, the problem is much more difficult, as classical abrupt change detection methods are ill-suited for detecting smooth 
changes in the system signals, especially in case of low amplitude oscillatory failures and noisy measurements.

A possible solution to this problem and the principal contribution of this work is to transform the sinusoidal influence of the COFC in the system into a step-like influence. The determination of the detection threshold is simplified and is solely based on the parameters of the measurement noise, usually available and depending on sensors performance. This transformation is obtained using an appropriate additional filter. From this perspective, the work reported in [4] will constitute the basis of the residual generator. In the original paper, an estimator is proposed to provide accurate estimation for a sinusoidal component in a noisy signal. It is shown that the outputs of the estimator converge asymptotically to the correct values. The core of the estimator is a third order nonlinear filter described by the relations:

$$
\begin{aligned}
& \dot{x}_{1}=K x_{3}\left(-2 \alpha\left[-2 \alpha x_{3}-\alpha^{2} x_{2}+u\right]-\alpha^{2} x_{3}\right) \\
& \quad-K x_{3}^{2} y-K\left[-2 \alpha x_{3}-\alpha^{2} x_{2}+u\right] u ; \\
& \dot{x}_{2}=x_{3} \\
& \dot{x}_{3}=-2 \alpha x_{3}-\alpha^{2} x_{2}+u \\
& y=x_{1}+K x_{3} u
\end{aligned}
$$

The coefficients $K$ and $\alpha$ are the tuning parameters, with $K$ acting like a gain and $\alpha$ acting like a damping ratio. When $t \rightarrow \infty$, the relation $\hat{\omega}_{0}=\sqrt{|y|}$ is an accurate estimation of the pulsation of an input oscillatory signal from the form 1 . The remaining parameters (amplitude and phase) are given by

$$
a=\sqrt{\frac{-\hat{\dot{u}}^{2}}{y}+\beta^{2}} ; \quad \phi=y-\beta
$$

with

$$
\begin{aligned}
& \beta=\frac{\hat{\ddot{u}}}{y} \\
& \hat{\dot{u}}=y x_{3}+2 \alpha\left[-2 \alpha x_{3}-\alpha^{2} x_{2}+u\right]+\alpha^{2} x_{3} \\
& \hat{\ddot{u}}=y\left[-2 \alpha x_{3}-\alpha^{2} x_{2}+u\right]+2 \alpha y x_{3}+\alpha^{2}\left[-2 \alpha x_{3}-\alpha^{2} x_{2}+u\right] .
\end{aligned}
$$

Here, $\hat{\dot{u}}$ and $\hat{\ddot{u}}$ are the input derivative estimates. The residual generator proposed in this work is thus based on the nonlinear filter described in (4). The filter is sensitive to the input of an oscillatory signal and reacts by a slope change of the output $y$. The proof is ommited for the sake of brevity (see [4]). 


\subsubsection{Residual evaluation}

The residual evaluation is a decision-making process which usually comes down to a threshold logic of a decision function [18]. Due to measurement noises, modeling uncertainties, robust residual evaluation is the only way to keep the false alarm rate small with an acceptable sensitivity to faults. Classically, robust residual evaluation can be accomplished in many ways, for example, by statistical data processing, data reconciliation, correlation, pattern recognition, fuzzy logic, or adaptive thresholds. In the considered case, the harmonic filter provides a good discrimination for all nonperiodic outputs and behaves in easily predictable way when a periodic signal is present in the processed data. Using the residual (or the energy of the residual) provided by the relations (4) and (3), the detection is improved using a discrete-time high gain observer proposed in [19]. The discrete transfer function is given below:

$$
T(z)=\frac{2 \beta}{2 \epsilon+\beta \Delta T} \frac{z-1}{z+(1-2 \epsilon /(\beta \Delta T)) /(1+2 \epsilon /(\beta \Delta T))}
$$

where $\Delta T$ is the sampling time and $\beta$ and $\epsilon$ are the tuning parameters to optimize false alarm / missed detection ratio. The behavior of this filter is fixed by the ratio $\Delta T / \epsilon$. High values (superior to 1 ) are taken when the noise is weak and low values when the noise is strong.

\section{SIMULATION RESULTS}

\subsection{Protocol}

In ADDSAFE project context [5], the simulations are performed using a high fidelity commercial aircraft benchmark under Matlab/Simulink environment. The measured parameter is the angle of attack. The measurement noise is a Gaussian noise with a $10^{-4}$ variance. For confidentiality concerns, all values are normalized. The sampling rate is $\Delta t=0.01 \mathrm{~s}$. Two cases are considered:

(1) a unique failure occurring at $t_{\text {def }}=6 \mathrm{~s}$ on the first sensor with all units initially being healthy. The detection and the isolation of the COFC is performed using soft monitoring module; and

(2) a second failure occurring at $t_{\text {def }}=9 \mathrm{~s}$ on the second sensor with the first unit initially offline. The detection and the isolation of the COFC are based on the analysis of the residuals corresponding to each source. 
Table 2 Combined oscillatory failure cases parameters

\begin{tabular}{cc}
\hline Frequency, $\mathrm{Hz}$ & Amplitude \\
\hline $0.2 ; 0.7 ; 5 ; 10$ & $0.5^{\circ} ; 1^{\circ}$ \\
\hline
\end{tabular}

Note that the second case where only two valid sources are available is very similar to the case when only one valid source exists, since the FDI method proposed in this work does not use data from other sources. For each case, simulations are performed with $\mathrm{COFC}$ of different frequencies and amplitudes (Table 2).

The tuning parameters of the Kalman filter are $\omega_{0}=4 \pi=2 \mathrm{~Hz}$ and $\xi=0.9$. The parameters of the harmonic filter are set to $K=20$ and $\alpha=10$. The parameter $\omega_{0}$ is taken near the middle of the frequency band of the expected OFCs, i. e., (0.1-10) Hz. The parameters $\xi, K$, and $\alpha$ are chosen using a Paretooptimum approach, maximizing fault detection ration and minimizing detection delay, missed detections and false alarms (to have an idea on the selection proce-

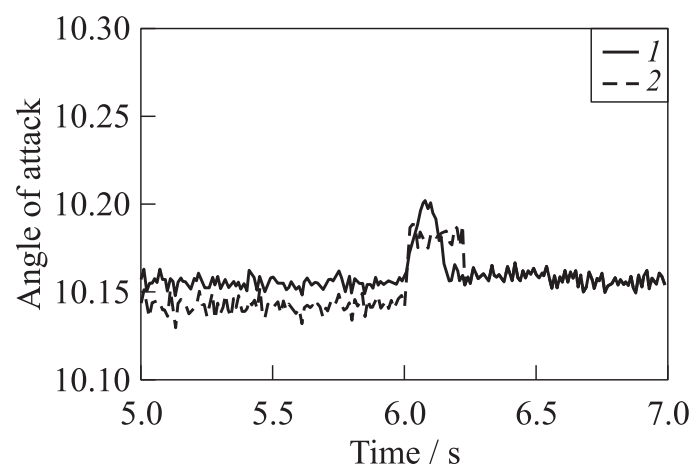

(a)

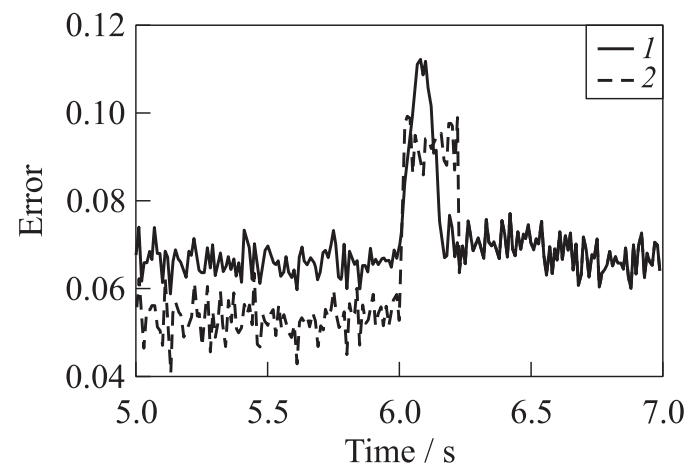

(b)

Figure 7 Soft monitoring simulation results: (a) measurement consolidation; (b) consolidation error; 1 - soft-computing; and 2 - classical 
dure, see [11]). The values are given for a COFC of amplitude $0.5^{\circ}$ and frequency of $0.7 \mathrm{~Hz}$.

For the robust derivative estimator, $\beta$ is set to 1 . If the measurement noise is weak, one can take $\Delta T / \epsilon=10$, but for the considered application, the ratio is fixed to 0.3 to obtain the best detection/false alarm ratio. This value is obtained using the approach from the previous paragraph given in [11].

\subsection{Unique Failure Scenario}

Figure 7 shows the simulation results for the COFC case $\left(a=0.5^{\circ}\right.$ and $f$ $=0.7 \mathrm{~Hz})$. Soft-computing based FDI scheme and classical voting approach are compared. It is easy to notice the discontinuity in the consolidated measurement obtained by the classical vote-based method, while the

Table 3 Soft-monitoring detection delays (in seconds)

\begin{tabular}{ccccc}
\hline \multirow{2}{*}{ Amplitude } & \multicolumn{4}{c}{ Frequency, $\mathrm{Hz}$} \\
\cline { 2 - 5 } & 0.2 & 0.7 & 5 & 10 \\
\hline $0.5^{\circ}$ & 0.63 & 0.23 & 0.13 & 0.17 \\
$1.0^{\circ}$ & 0.33 & 0.13 & 0.07 & 0.07 \\
\hline
\end{tabular}
soft-computing based consolidated measurement remains smooth. Parameter discontinuities can lead to unwanted controller behaviors and should be avoided. The remaining COFC case results are processed likewise. The detection delays are given in Table 3. The delays are given as the difference between COFC occurrence in the system and COFC detection by the implemented FDI method.

\subsection{Double Failure Scenario}

When two simultaneous OFC occur on the first and the second sensors, the consolidation module will perform detection but fails to switch off the faulty sources due to majority-vote principle (see section 2). However, the results given in Table 4 let to notice that fault isolation is successfully carried by the Table 4 Failure detection and isolation delays (in seconds)

\begin{tabular}{ccccc}
\hline \multirow{2}{*}{ Amplitude } & \multicolumn{4}{c}{ Frequency, $\mathrm{Hz}$} \\
\cline { 2 - 5 } & 0.2 & 0.7 & 5 & 10 \\
\hline $0.5^{\circ}$ & 0.4 & 0.29 & 0.23 & 0.23 \\
$1.0^{\circ}$ & 0.37 & 0.25 & 0.22 & 0.22 \\
\hline
\end{tabular}
implemented FDI approach for all fault frequencies. Hence, any possible wrong decisions could be corrected. Figure 8 shows the simulation results for the COFC case $\left(a=0.5^{\circ}\right.$ and $\left.f=0.7 \mathrm{~Hz}\right)$. The residual reacts as expected to the occurrence of the failure. 


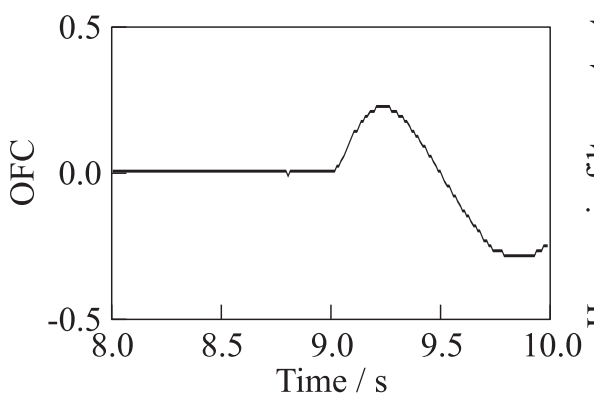

(a)

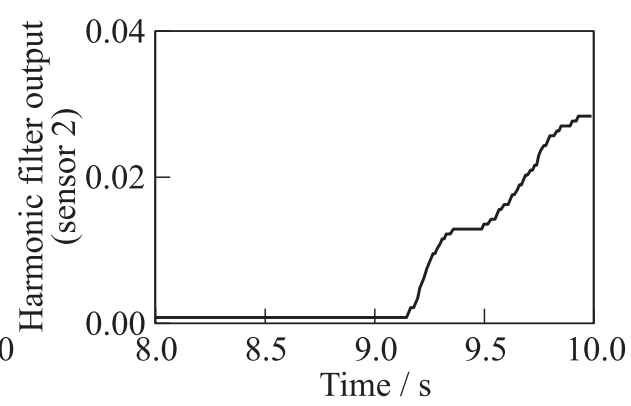

(b)

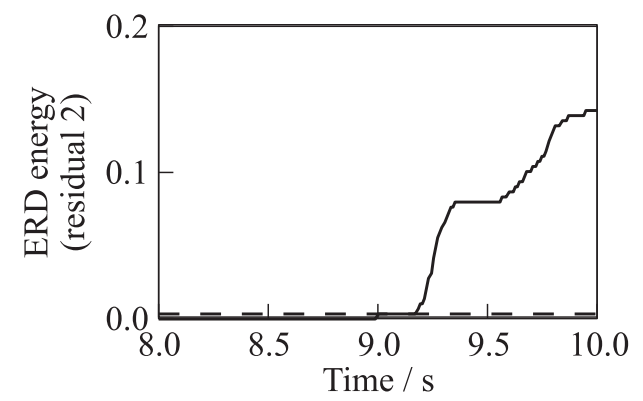

(c)

Figure 8 Combined oscillatory failure detection and isolation cases based on residuals (ERD - equivalent residual dose)

\subsection{Discussion}

Simulation results show the complementarity of the two monitoring approaches. When all the flight parameter sensors are initially healthy, soft-monitoring successfully detection and switches off the corrupted source, with best performance achieved for frequencies superior to $0.2 \mathrm{~Hz}$. When the soft-monitoring is not suitable, FDI based on residual generation successfully detects COFC failures for all frequencies compared to the work investigated in [11].

\section{CONCLUDING REMARKS}

An approach to oscillatory FDI in aircraft air data/inertial system is presented. Fuzzy logic approach for consolidation is combined with signal processing based FDI to extend the conventional scheme in order to manage a low number of healthy sensors. The proposed approach was successfully implemented on a high 
fidelity commercial aircraft benchmark, and the simulation results confirm efficiency of the combined FDI method vs. the conventional scheme.

\section{ACKNOWLEDGMENTS}

This work was performed in the framework of the European ADDSAFE project: Grant agreement No. FP7-233815.

\section{REFERENCES}

1. Jia, H. 2004. Data fusion methodologies for multisensor aircraft navigation systems. Ph.D. Cranfield University.

2. Oosterom, M., R. Babuska, and H. Verbruggen. 2002. Soft computing applications in aircraft sensor management and flight control law reconfiguration. IEEE Trans. Syst. Man Cybernetics, Part C (Applications and Reviews) 32(2):125-39.

3. Hegg, J. 2002. Enhanced space integrated GPS/INS (SIGI). IEEE Aerospace Electronic Syst. J. 17(4):26-33.

4. Aranovskiy, S., A. Bobtsov, A. Kremlev, N. Nikolaev, and O. Slita. 2007. Identification of frequency of biased harmonic signal. 9th IFAC Workshop - Adaptation and Learning in Control and Signal Processing.

5. http://addsafe.deimos-space.com/.

6. Goupil, P. 2009. Oscillatory failure case detection in the A380 electrical flight control system by analytical redundancy. Control Engineering Practice 18(9):1110-19.

7. Alcorta Garcia, E., A. Zolghadri, and G. Philippe. 2009. A novel non-linear observer-based approach to oscillatory failure detection. European Control Conference ECC'09 Proceedings. Budapest, Hungary. 1901-6.

8. Rosenberg, K. 1988. FCS architecture definition (issue 1) Deliverable 3.4. BE974098.

9. Osder, S. 1999. Practical view of redundancy management application and theory. J. Guidance Control Dyn. 22(1).

10. Goupil, P. 2009. Airbus state of the art and practices on FDI and FTC. 7th IFAC Symposium on Fault Detection, Supervision and Safety of Technical Processes Proceedings. Barcelona, Spain.

11. Berdjag, D., A. Zolghadri, J. Cieslak, and P. Goupil. 2010. Fault detection and isolation for redundant aircraft sensors. IEEE Conference on Control and Fault Tolerant Systems.

12. Middleton, R., and G. Goodwin. 1990. Digital control and estimation. Ed. T. Kailath. Englewood Cliffs, New Jersey: Prentice Hall, Inc.

13. Zolghadri, A. 1996. An algorithm for real-time failure detection in Kalman filters. IEEE Trans. Automatic Control 41(10):1537-39. 
14. Basseville, M., and I. V. Nikiforov. 1993. Detection of abrupt changes - theory and application. Englewood Cliffs, N.J.: Prentice-Hall, Inc.

15. Patton, R. J. 1994. Robust model-based fault diagnosis: The state of the art. IFAC Symposium SAFEPROCESS'94 Proceedings. Espoo, Finland. 1:1-24.

16. Isermann, R. 2005. Model-based filt detection and analysis - status and application. Ann. Rev. Control 29:71-85.

17. Marzat, J., H. Piet-Lahanier, F. Damongeot, and E. Walter. 2009. A new modelfree method performing closed-loop fault diagnosis for an aeronautical system. 7 th Workshop on Advanced Control and Diagnosis, ACD'2009. Zielona Gora, Poland.

18. Frank, P., and X. Ding. 1997. Survey of robust residual generation and evaluation methods in observer-based fault detection systems. J. Process Control 7(6):403-24.

19. Dabroom, A., and H. Khalil. 1999. Discrete-time implementation of high-gain observers for numerical differentiation. Int. J. Control 72(17):1523-37. 